Research, Society and Development, v. 9, n.12, e46091211501, 2020

(CC BY 4.0) | ISSN 2525-3409 | DOI: http://dx.doi.org/10.33448/rsd-v9i12.11501

\title{
Reacción de cuerpo extraño en maxilar superior: reporte de caso
}

Foreign body reaction in maxilla: case report

Reação de corpo estranho na maxila: relato de caso

Recibido: 25/12/2020 | Revisado: 26/12/2020 | Acepto: 27/12/2020 | Publicado: 29/12/2020

Gabriela Nathaly Berrezueta Arízaga

ORCID: https://orcid.org/0000-0003-4260-0779

Universidad de Cuenca, Ecuador

E-mail: gabriela.berrezueta@ucuenca.edu.ec

David Manuel Pineda Álvarez

ORCID: https://orcid.org/0000-0002-6395-7702

Universidad de Cuenca, Ecuador

E-mail: david.pineda@ucuenca.edu.ec

\section{Resumen}

La reacción de cuerpo extraño es una respuesta inmunitaria que consiste en un estado inflamatorio persistente en el cual un dispositivo, prótesis o biomaterial es rechazado por el organismo, lo cual induce a su fagocitosis o degradación sin éxito. Luego de este proceso frustrado de eliminación provoca que los macrófagos se fusionen formando células gigantes de cuerpo extraño, y que tras el acúmulo de colágeno secretado por fibroblastos se dé la formación de una cápsula fibrosa que aísla al biomaterial del medio tisular. El tratamiento de esta reacción consiste en la eliminación quirúrgica de la lesión con la posterior regeneración del defecto, constituyendo los injertos y sustitutos óseos como la mejor opción terapéutica y destacando entre estos tanto autoinjertos como aloinjertos. En la presente revisión de la literatura se presenta el caso clínico de una reacción de cuerpo extraño en el maxilar superior con sus características clínicas, radiográficas, tratamiento y control clínico postoperatorio.

Palabras clave: Reacción de cuerpo extraño; Substitutos óseos; Aloinjertos; Autoinjertos.

\section{Abstract}

The foreign body reaction is an immune response that consists of a persistent inflammatory state in which a device, prosthesis, or biomaterial is rejected by the body, which induces its phagocytosis or unsuccessful degradation. After this frustrated elimination process, it causes the macrophages to fuse to form giant foreign body cells, and after the accumulation of 
collagen secreted by fibroblasts, the formation of a fibrous capsule occurs that isolates the biomaterial from the tissue medium. The treatment of this reaction consists of the surgical removal of the lesion with the subsequent regeneration of the defect, constituting the grafts and bone substitutes as the best therapeutic option and highlighting among these both autografts and allografts. In this review of the literature, the clinical case of a foreign body reaction in the maxilla is presented with its clinical and radiographic characteristics, treatment and postoperative clinical control.

Keywords: Foreign-body reaction; Bone substitutes; Allografts; Autografts.

\section{Resumo}

A reação de corpo estranho é uma resposta imune que consiste em um estado inflamatório persistente no qual um dispositivo, prótese ou biomaterial é rejeitado pelo corpo, o que induz sua fagocitose ou degradação sem sucesso. Após esse frustrado processo de eliminação, faz com que os macrófagos se fundam para formar células gigantes de corpo estranho e, após o acúmulo de colágeno secretado pelos fibroblastos, ocorre a formação de uma cápsula fibrosa que isola o biomaterial do meio tecidual. $O$ tratamento dessa reação consiste na retirada cirúrgica da lesão com a posterior regeneração do defeito, constituindo os enxertos e substitutos ósseos como a melhor opção terapêutica e destacando-se entre os autoenxertos e aloenxertos. Nesta revisão da literatura, é apresentado o caso clínico de reação de corpo estranho em maxilar superior com suas características clínicas e radiográficas, tratamento e controle clínico pós-operatório.

Palavras-chave: Reação de corpo estranho; Substitutos ósseos; Aloenxertos; Autoenxertos.

\section{Introducción}

La reacción de cuerpo extraño es una respuesta inflamatoria crónica que se produce cuando un dispositivo, prótesis o biomaterial es reconocido por el organismo como patógeno y provoca reacciones inmunitarias en las cuales este no puede ser degradado, provocando así una destrucción de los tejidos y su aislamiento o rechazo (Anderson et al., 2008; Klopfleisch \& Jung, 2017; Mariani et al., 2019).

Los biomateriales inducen procesos celulares y tisulares que provocan respuestas inflamatorias y de cicatrización, así como también reacciones de cuerpo extraño o el encapsulamiento de materiales (Sheikh et al., 2015); si bien todos deben tener capacidad de proveer soportes biocompatibles, debido a sus múltiples compuestos cada uno tendrá distintas 
funciones y características estructurales, por lo cual la interacción con el ambiente biológico y la respuesta pro o antiinflamatoria dependerá del tejido sobre el que actúee influirá en el reclutamiento y adhesión de los tejidos(Anderson et al., 2008) lo cual impulsará la activación del sistema inmune innato y por consiguiente el inicio de la respuesta adaptativa (Anderson et al., 2008; Klopfleisch \& Jung, 2017; Mariani et al., 2019).

Existen múltiples eventos que se desarrollarán simultáneamente:

\section{Adsorción de proteínas}

Tras la implantación, las proteínas que se encuentran presentes en el plasma sanguíneo se adsorben en la superficie del material conformando una matriz provisional (Anderson et al., 2008; Kastellorizios et al., 2015; Klopfleisch \& Jung, 2017; Mariani et al., 2019; Rolvien et al., 2018; Sheikh et al., 2015), la cual posee capacidad para liberar factores de crecimiento, citocinas y quimioatrayentes(Anderson et al., 2008; Klopfleisch \& Jung, 2017; Sheikh et al., 2015). La albúmina, fibronectina y vitronectina son proteínas importantes en este proceso, siendo las dos últimas junto con los agentes bioactivos liberados fundamentales en la adhesión de monocitos y formación de células gigantes(Anderson et al., 2008; Kastellorizios et al., 2015; Klopfleisch \& Jung, 2017; Mariani et al., 2019). Tras este proceso se obtendrá una matriz final conformada por un coágulo de fibrina(Klopfleisch \& Jung, 2017).

\section{Inflamación Aguda}

El sistema de complemento se activa debido a la interacción entre la sangre y el material con lo cual se inicia la respuesta innata del organismo produciendo la infiltración de glóbulos blancos(Anderson et al., 2008; Kastellorizios et al., 2015; Klopfleisch \& Jung, 2017; Rolvien et al., 2018; Sheikh et al., 2015). Patrones moleculares asociados a daño (DAMP) son liberados tras la ruptura de las membranas celulares y son reconocidos por PMN, lo cual provoca su migración hacia el lugar de implantación(Klopfleisch \& Jung, 2017; Mariani et al., 2019). Tras su llegada y adhesión, tanto PMN como neutrófilos son activados y degranulados, la histamina liberada atrae más leucocitos con lo cual se empieza a establecer la inflamación crónica(Anderson et al., 2008; Kastellorizios et al., 2015; Klopfleisch \& Jung, 2017). 


\section{Inflamación crónica}

Antes y durante esta fase monocitos y linfocitos circulantes responden a plaquetas, PMN y quimioatrayentes(Klopfleisch \& Jung, 2017); los macrófagos son atraídos por factores de crecimiento, de complemento y proteínas inflamatorias hacia el sitio de implantación en el cual se adhieren principalmente al fibrinógeno, vitronectina y fibronectina, unión que produce su activación. Estos permanecen en el lugar tratando de fagocitar el material extraño y reclutar otras células como fibroblastos(Anderson et al., 2008; Kastellorizios et al., 2015; Klopfleisch \& Jung, 2017; Rolvien et al., 2018; Sheikh et al., 2015).

\section{Formación de Células gigantes de cuerpo extraño}

Después de un proceso de fagocitosis frustrada se produce la fusión de macrófagos, los cuales conformarán células gigantes de cuerpo extraño con el objetivo de mejorar su eficacia(Anderson et al., 2008; Kastellorizios et al., 2015; Klopfleisch \& Jung, 2017; Rolvien et al., 2018; Sheikh et al., 2015).

\section{Formación de cápsulas fibrosas}

Fibroblastos y células endoteliales son atraídas hacia la superficie del material, sobre este depositan colágeno y otras proteínas para la formación de tejido de granulación que posteriormente se hará más fibroso, denso y menos celular llevando a que se encapsule y aísle del medio tisular(Anderson et al., 2008; Kastellorizios et al., 2015; Klopfleisch \& Jung, 2017; Rolvien et al., 2018; Sheikh et al., 2015). En ocasiones los fibroblastos se diferencian en miofibroblastos, lo cual puede producir una contracción de la cápsula(Klopfleisch \& Jung, 2017; Rolvien et al., 2018). El proceso cicatrizal no se lleva a cabo debido a la persistencia del agente iniciador, el biomaterial y el proinflamatorio continuo(Klopfleisch \& Jung, 2017).

Cuando una reacción de cuerpo extraño es tratada quirúrgicamente se requiere la regeneración tisular de los defectos existentes, para lo cual está indicado el uso de injertos o sustitutos óseos(Fillingham \& Jacobs, 2016; García-Gareta et al., 2015; Jordana et al., 2017; Miron, Sculean, et al., 2016); los cuales deben tener integridad natural y capacidad para promover el reclutamiento de células con potencial de formación ósea(Deluiz et al., 2017). El material ideal debe ser osteogénico, osteoinductivo, y osteoconductor(Deluiz et al., 2017; Fillingham \& Jacobs, 2016; Miron, Sculean, et al., 2016) es decir, tener capacidad de formar 
matriz nueva, reclutar e inducir la diferenciación de células mesenquimatosas para producir hueso maduro y permitir la invasión vascular e infiltración celular(Miron, Sculean, et al., 2016).

Existen múltiples tipos de injertos y sustitutos, los cuales variarán en su composición, características y tasa de éxito. En general, el injerto autólogo es el "gold standard" por lo cual se lo considera la mejor opción de tratamiento ya que proviene del mismo individuo(Deluiz et al., 2017; Fillingham \& Jacobs, 2016; García-Gareta et al., 2015; Haugen et al., 2019; Jordana et al., 2017; Miron, Sculean, et al., 2016; Miron, Zhang, et al., 2016; Papageorgiou et al., 2016), puede obtenerse de sitios donantes intra o extraorales(Nissan et al., 2018), tiene excelentes propiedades e histocompatibilidad completa(Fillingham \& Jacobs, 2016), no obstante, su alto costo, suministro limitado, transmisión de virus, reabsorción impredecible y morbilidad postquirúrgica han hecho que los profesionales opten por otras opciones terapéuticas(Deluiz et al., 2017; Fillingham \& Jacobs, 2016; García-Gareta et al., 2015; Haugen et al., 2019; Miron, Zhang, et al., 2016; Nissan et al., 2018, p.; Papageorgiou et al., 2016) entre las que podemos encontrar tres grupos: aloinjertos, xenoinjertos y sustitutos óseos sintéticos(Fillingham \& Jacobs, 2016; Papageorgiou et al., 2016).

El injerto óseo homólogo o también denominado como aloinjerto es muy utilizado(Haugen et al., 2019), proviene de otro individuo de la misma especie(García-Gareta et al., 2015; Haugen et al., 2019), se obtiene por lo general de la pelvis de cadáveres(Fillingham \& Jacobs, 2016; García-Gareta et al., 2015; Jordana et al., 2017) y puede adquirirse en bancos de hueso(Haugen et al., 2019; Jordana et al., 2017), es principalmente osteoconductor y tiene características osteoinductoras reducidas(GarcíaGareta et al., 2015; Haugen et al., 2019). Si bien no es osteogénico, la gran cantidad disponible, su bajo costo y no producir morbilidad forman parte de sus ventajas, sin embargo puede darse una curación incompleta(Fillingham \& Jacobs, 2016) y existe riesgo de inmunoreacciones e infecciones (Fillingham \& Jacobs, 2016; García-Gareta et al., 2015; Haugen et al., 2019).

Los xenoinjertos son materiales óseos naturales provenientes de otra especie, generalmente de bovinos(Haugen et al., 2019; Jordana et al., 2017; Miron, Zhang, et al., 2016), tienen riesgo de transmitir enfermedades infecciosas, producir respuestas inmunes en el receptor y su tasa de reabsorción es variable(Haugen et al., 2019; Jordana et al., 2017).

Los injertos sintéticos buscan imitar la composición del hueso para poder regenerar los defectos sin embargo esto se dificulta debido a su osteoinductividad limitada. Dentro de este 
grupo se pueden mencionar a los biopolímeros, cerámicas, entre otros(García-Gareta et al., 2015; Jordana et al., 2017).

Junto al material de regeneración ósea se suele utilizar plasma rico en fibrina (FRP) el cual es un aditivo quirúrgico bioactivo obtenido del mismo paciente, se caracteriza por su alto potencial regenerativo y sus propiedades biológicas ya que libera factores de crecimiento, estimula la angiogénesis, mejora la cicatrización, es de fácil obtención y costo bajo(Feigin \& Shope, 2019; Mohan et al., 2019; Shah et al., 2019). El protocolo convencional de elaboración de este autoinjerto se basa en centrifugar sangre venosa sin aditivos en tubos de ensayo de 10 ml recubiertos de sílice a $2700 \mathrm{rpm}$ por 10 a 12 minutos(Mohan et al., 2019; Shah et al., 2019; Sohn et al., 2015).

A partir de este proceso se forman 3 capas distintas, la primera con glóbulos rojos, la segunda a manera de coágulo, en la cual existe fibrina polimerizada, glóbulos blancos y glucosaminoglicanos, y la última que es pobre en plaquetas. La capa intermedia es la que se aprovecha para la formación de membranas(Mohan et al., 2019; Shah et al., 2019; Sohn et al., 2015).

Al mezclar plasma rico en fibrina líquida (I- PRF) e injerto óseo particulado se obtiene un injerto óseo estable de fibrina o también denominado como hueso adhesivo (sticky bone), el cual es moldeable debido a que posee una fuerte red de interconexión de fibrina que le da estabilidad sobre el defecto previniendo así que se den macro o micro movimientos. Esta combinación permite la liberación de factores de crecimiento provenientes de leucocitos, mejora la regeneración de tejidos óseos y blandos y minimiza la pérdida de hueso durante el periodo de curación (Sohn et al., 2015; Soni, Priya, Kumar, et al., 2019; Soni, Priya, Yadav, et al., 2019).

\section{Metodología}

El enfoque metodológico del presente artículo consiste en el reporte de un caso clínico, más no en una investigación. La publicación cumple con los principios éticos, puesto que, para llevar a cabo este estudio, el paciente firmó el consentimiento informado de la intervención quirúrgica, así como la aprobación para su publicación; en todo momento se respeta el anonimato del paciente. Para su desarrollo no se requirió la aplicación de encuestas, puesto que se contó con una detallada historia clínica (Pereira et al., 2018)

Los autores se autofinancian y tienen el apoyo docente de la Facultad de Odontología de la Universidad de Cuenca. 


\subsection{Presentación de caso clínico}

Paciente de sexo femenino de 25 años de edad asiste a la consulta odontológica general debido a asimetría facial dependiente de lado derecho, la cual se presenta a manera de un abultamiento y enrojecimiento a nivel de la piel de la región, por dentro del surco vestibular la paciente evidencia una lesión indurada, expuesta a la cavidad oral, por encima de canino y lateral, con encía enrojecida y dolorosa alrededor. Tras exploración clínica intraoral se observa una lesión blanquecina con secreción de tipo purulenta a su alrededor, firme, contextura rígida similar al hueso y diámetro aproximado de $1,5 \mathrm{~cm}$. (Figura 1). Debido a las características de la lesión, se refiere el caso al especialista maxilofacial.

Figura 1. Imagen clínica de la lesión.

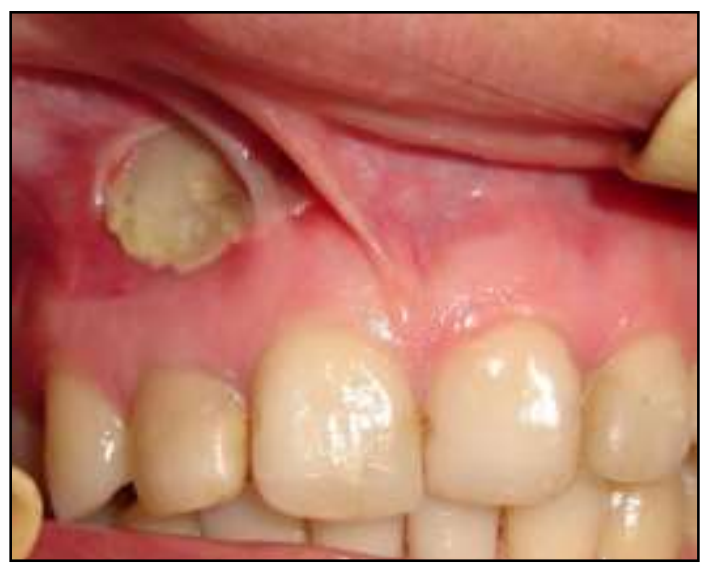

Fuente: Dr. Juan Carlos Terán.

Durante la anamnesis la paciente manifiesta que hace cuatro meses aproximadamente le realizaron tratamiento endodóntico en las piezas 1.1, 1.2, 1.3 y apicectomía de las mismas debido a la presencia de un quiste. Posteriormente le colocaron un material de regeneración ósea, el cual desconoce. No refiere antecedentes patológicos personales ni familiares y los exámenes complementarios solicitados tuvieron valores normales, a excepción del hemograma en el cual se evidenció una leucocitosis con neutrofilia.

Durante el análisis radiográfico se observó que a nivel apical de las piezas 1.1, 1.2, 1.3 existía un área radiopaca con halo radiolúcido y bordes definidos abarcando el sector anterior del primer cuadrante del hueso maxilar, en relación con los ápices dentarios de las piezas mencionadas (Figura 2). 
Figura 2. Radiografía periapical de la lesión.

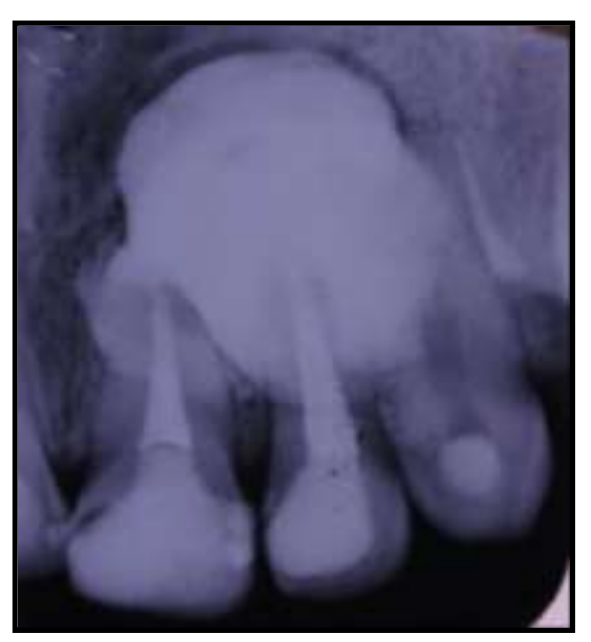

Fuente: Dr. Juan Carlos Terán.

\section{Intervención quirúrgica}

Se programó una biopsia exploratoria para la eliminación de la lesión, el procedimiento se realizó mediante anestesia local, la lesión fue extraída con facilidad en un solo bloque de $2.5 \mathrm{~cm}$ de diámetro con fragmentos residuales que posteriormente fueron limpiados (Figura 3); alrededor se presentó tejido blando de tipo granulomatoso el cual se eliminó mediante curetaje dejando hueso sano y sangrante (Figura 4). Para la regeneración ósea de la cavidad dejada tras la extracción de la lesión se colocó hueso adhesivo (Sticky bone) formado a partir de la mezcla de aproximadamente $3.5 \mathrm{cc}$ de aloinjerto particulado junto con PRF (Figura 5) y se colocó una membrana de fibrina como cobertura, con posterior sutura. 
Figura 3. Escisión quirúrgica de la lesión.

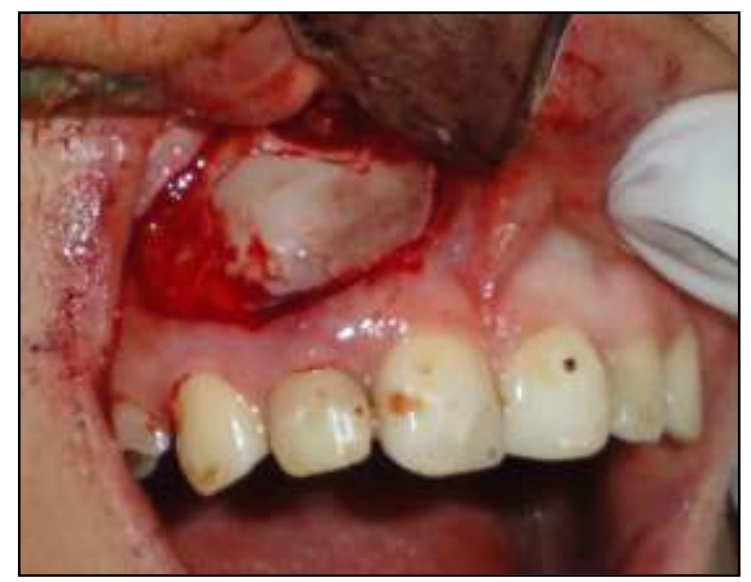

Fuente: Dr. Juan Carlos Terán.
Figura 4. Lecho quirúrgico.

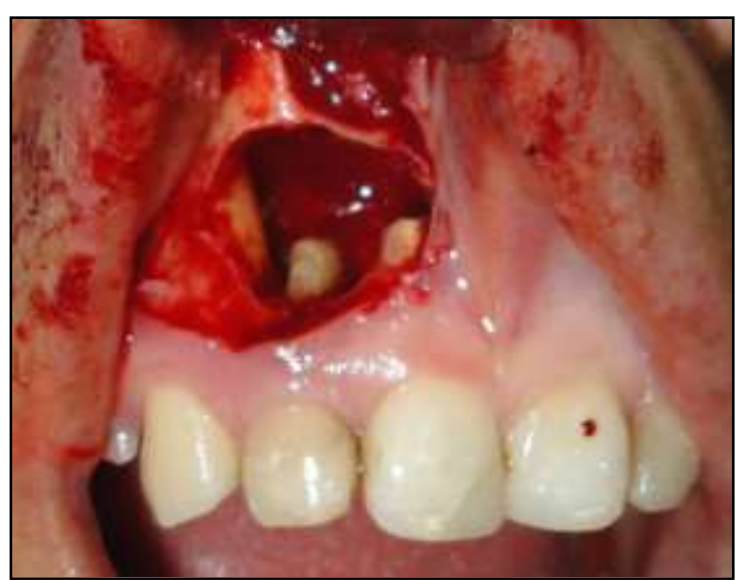

Fuente: Dr. Juan Carlos Terán.

Figura 5. Relleno del defecto óseo con sticky bone.

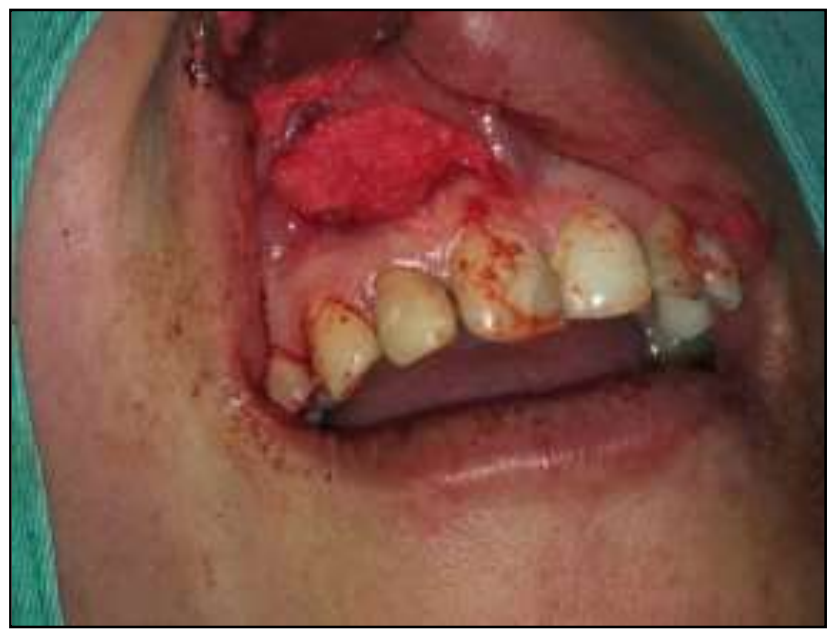

Fuente: Dr. Juan Carlos Terán.

No se realizó examen histopatológico debido a que tras el análisis clínico al cortar el cuerpo extraño con una fresa quirúrgica este evidenció olor, contextura y color, compatible con material de restauración dental (composite).

El control se realizó a los 8 días y al mes de la intervención quirúrgica. En el primer control durante la exploración clínica intraoral se observó la dehiscencia de puntos de sutura y en el segundo ya existió un cierre completo de la lesión con una cicatrización completa (Figuras 6 y 7 ). 
Figuras 6 y 7. Controles clínicos postquirúrgicos.

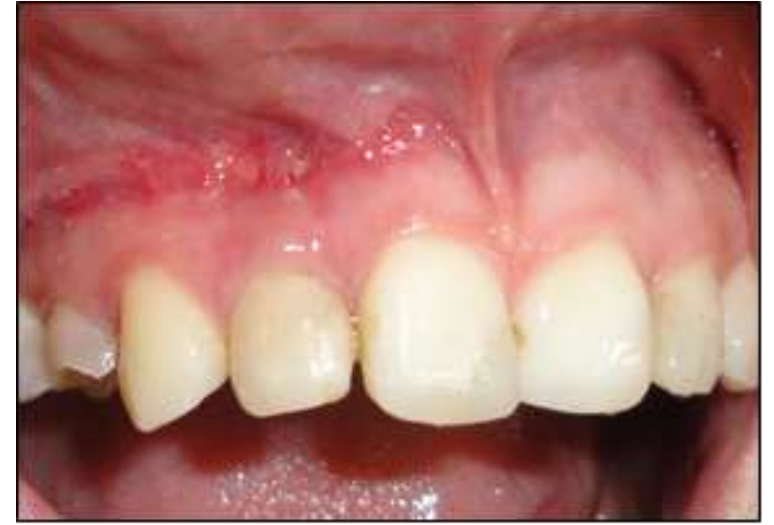

Fuente: Dr. Juan Carlos Terán.

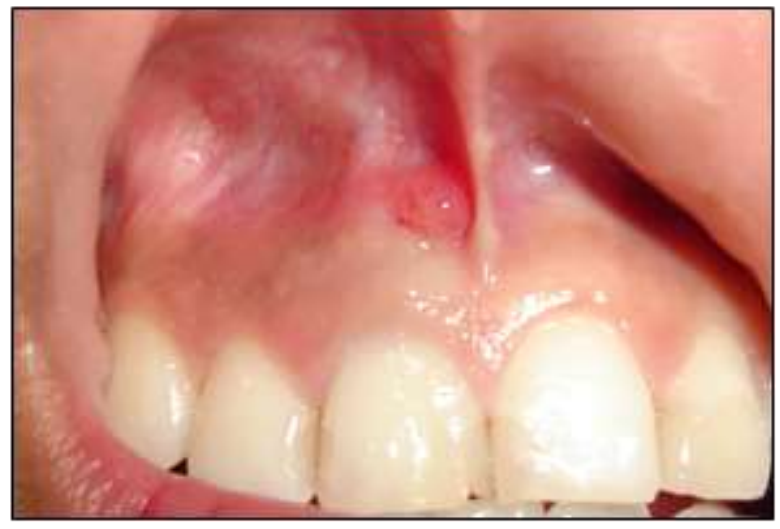

Fuente: Dr. Juan Carlos Terán.

\section{Resultados y Discusión}

Los biomateriales y sustitutos óseos producen respuestas inflamatorias específicas en los diferentes tejidos(Anderson et al., 2008; Klopfleisch \& Jung, 2017; Mariani et al., 2019; Rolvien et al., 2018; Sheikh et al., 2015), las cuales dependen de las características superficiales y propiedades fisicoquímicas del agente reconocido como patógeno, estas determinarán la gresolución del proceso inflamatorio o por el contrario el inicio de una cascada denominada "reacción de cuerpo extraño"(Anderson et al., 2008; Kastellorizios et al., 2015; Klopfleisch \& Jung, 2017; Mariani et al., 2019; Rolvien et al., 2018; Sheikh et al., 2015). Macrófagos, monocitos y neutrófilos interactúan en los tejidos y tienen vital importancia en el éxito curativo ya que actúan como reguladores en el proceso de degradación y determinan la respuesta pro o antiinflamatoria(Anderson et al., 2008; Kastellorizios et al., 2015; Klopfleisch \& Jung, 2017; Mariani et al., 2019; Rolvien et al., 2018).

El tener una reacción biológica, química y mecánica adecuada dependerá tanto de la biocompatibilidad del material (Anderson et al., 2008; Mariani et al., 2019), así como de su correcto uso por parte del profesional (Jordana et al., 2017; Klopfleisch \& Jung, 2017; Rolvien et al., 2018). Si bien todos los materiales que se utilizan dentro de la consulta odontológica deben ser biocompatibles, es decir cumplir una función y reacción determinada sin generar efectos locales o sistémicos, se debe conocer las limitantes de cada uno (Mariani et al., 2019; Rolvien et al., 2018), ya que pueden tener una respuesta favorable o por el contrario resultar lesivos (Klopfleisch \& Jung, 2017; Rolvien et al., 2018). Se debe recordar que no todo material de restauración dental a pesar de ser biocompatible puede utilizarse como material de regeneración tisular. 
Para la regeneración ósea existen múltiples alternativas que variarán en sus características, propiedades, facilidad de adquisición y costo (García-Gareta et al., 2015; Haugen et al., 2019; Jordana et al., 2017). Ningún injerto disponible en la actualidad cumple todas las características requeridas(Jordana et al., 2017), por lo cual queda a criterio profesional el seleccionar la opción terapéutica adecuada de acuerdo al caso clínico a tratar (Fillingham \& Jacobs, 2016; Haugen et al., 2019; Jordana et al., 2017; Rolvien et al., 2018). Para este tratamiento se optó por el hueso adhesivo o sticky bone; la combinación de un autoinjerto (PRF) y un injerto óseo (hueso homólogo particulado) permitieron una óptima corrección de los defectos óseos y blandos, evidenciando a nivel clínico y radiográfico el éxito del procedimiento.

\section{Conclusiones}

-Los medios de defensa del organismo determinan la aceptación o el rechazo de un biomaterial por tanto su biocompatibilidad es fundamental pero no el único determinante para una adecuada respuesta biológica.

-El composite es un biomaterial odontológico cuya biocompatibilidad se limitada a su uso en los tejidos dentarios, por tanto, constituye un material de restauración dental mas no de restauración ósea.

-El autoinjerto es la opción más óptima para la regeneración ósea, sin embargo el aloinjerto es la alternativa más económica, accesible, bien aceptada por los tejidos y que además puede reparar defectos amplios sin producir morbilidad ni comprometer los tejidos sanos del paciente.

-El uso del injerto óseo estable de fibrina (Sticky bone) combina los beneficios del injerto óseo particulado y el I- PRF con lo cual se reduce el dolor e inflamación posterior a la cirugía, a la vez que mejora el proceso cicatrizal y regenerativo.

-Todo tratamiento requiere un control clínico y radiográfico a corto y largo plazo para prevenir el progreso de lesiones inflamatorias en procesos crónicos como las reacciones de cuerpo extraño.

En el presente artículo se puede concluir que, al no existir un consenso sobre el tratamiento de los defectos óseos originados por la eliminación quirúrgica de reacciones de cuerpo extraño, el especialista debe analizar minuciosamente la extensión de la lesión, edad, historia clínica y tipo de paciente con el fin de elegir la opción terapéutica más adecuada para 
cada caso. Se recomienda realizar más estudios clínicos comparativos para el tratamiento de reacciones de cuerpo extraño.

\section{Referencias}

Anderson, J. M., Rodriguez, A., \& Chang, D. T. (2008). Foreign body reaction to biomaterials. Seminars in Immunology, 20(2), 86-100. https://doi.org/10.10 16/j.smim.2007.11.004

Deluiz, D., Santos Oliveira, L., Ramôa Pires, F., Reiner, T., Armada, L., Nunes, M. A., \& Muniz Barretto Tinoco, E. (2017). Incorporation and remodeling of bone block allografts in the maxillary reconstruction: A randomized clinical trial. Clinical implant dentistry and related research, 19(1), 180-194.

Feigin, K., \& Shope, B. (2019). Use of Platelet-Rich Plasma and Platelet-Rich Fibrin in Dentistry and Oral Surgery: Introduction and Review of the Literature. Journal of Veterinary Dentistry, 36(2), 109-123. https://doi.org/10.1177/0898756419876057

Fillingham, Y., \& Jacobs, J. (2016). Bone grafts and their substitutes. The bone \& joint journal, 98(1_Supple_A), 6-9.

García-Gareta, E., Coathup, M. J., Blunn, G. W., \& Kumar, L. (2015). Osteoinduction of bone grafting materials for bone repair and regeneration. Bone, 81, 112-121. https://doi.org/10.1016/j.bone.2015.07.007

Haugen, H. J., Lyngstadaas, S. P., Rossi, F., \& Perale, G. (2019). Bone grafts: Which is the ideal biomaterial? Journal of Clinical Periodontology, 46, 92-102. https://doi.org/10.1111/jcpe.13058

Jordana, F., Le Visage, C., \& Weiss, P. (2017). Substituts osseux. médecine/sciences, 33(1), 60-65. https://doi.org/10.1051/medsci/20173301010

Kastellorizios, M., Tipnis, N., \& Burgess, D. J. (2015). Foreign Body Reaction to Subcutaneous Implants. En J. D. Lambris, K. N. Ekdahl, D. Ricklin, \& B. Nilsson (Eds.), 
Immune Responses to Biosurfaces, 865, 93-108. Springer International Publishing. https://doi.org/10.1007/978-3-319-18603-0_6

Klopfleisch, R., \& Jung, F. (2017). The pathology of the foreign body reaction against biomaterials: Foreign Body Reaction to Biomaterials. Journal of Biomedical Materials Research Part A, 105(3), 927-940. https://doi.org/10.1002/jbm.a.35958

Mariani, E., Lisignoli, G., Borzì, R. M., \& Pulsatelli, L. (2019). Biomaterials: Foreign Bodies or Tuners for the Immune Response? International Journal of Molecular Sciences, 20(3). https://doi.org/10.3390/ijms20030636

Miron, R. J., Sculean, A., Shuang, Y., Bosshardt, D. D., Gruber, R., Buser, D., Chandad, F., \& Zhang, Y. (2016). Osteoinductive potential of a novel biphasic calcium phosphate bone graft in comparison with autographs, xenografts, and DFDBA. Clinical Oral Implants Research, 27(6), 668-675. https://doi.org/10.1111/clr.12647

Miron, R. J., Zhang, Q., Sculean, A., Buser, D., Pippenger, B. E., Dard, M., Shirakata, Y., Chandad, F., \& Zhang, Y. (2016). Osteoinductive potential of 4 commonly employed bone grafts. Clinical Oral Investigations, 20(8), 2259-2265. https://doi.org/10.1007/s00784-016$1724-4$

Mohan, S. P., Jaishangar, N., Devy, S., Narayanan, A., Cherian, D., \& Madhavan, S. S. (2019). Platelet-Rich Plasma and Platelet-Rich Fibrin in Periodontal Regeneration: A Review. Journal of Pharmacy \& Bioallied Sciences, 11(Suppl 2), S126-S130. https://doi.org/10.4103/JPBS.JPBS_41_19

Nissan, J., Kolerman, R., Chaushu, L., Vered, M., Naishlos, S., \& Chaushu, G. (2018). Age-related new bone formation following the use of cancellous bone-block allografts for reconstruction of atrophic alveolar ridges. Clinical implant dentistry and related research, 20(1), 4-8.

Papageorgiou, S. N., Papageorgiou, P. N., Deschner, J., \& Götz, W. (2016). Comparative effectiveness of natural and synthetic bone grafts in oral and maxillofacial surgery prior to 
insertion of dental implants: Systematic review and network meta-analysis of parallel and cluster randomized controlled trials. Journal of Dentistry, 48, 1-8.

Pereira, A. S., Shitsuka, D. M., Parreira, F. J., \& Shitsuka, R. (2018). Metodologia da pesquisa científica. Brasil. https://repositorio.ufsm.br/ bitstream/handle/1/15824/Lic_Compu tacao_Metodologia-Pesquisa-Cientifica.pdf?se quence=1

Rolvien, T., Barbeck, M., Wenisch, S., Amling, M., \& Krause, M. (2018). Cellular mechanisms responsible for success and failure of bone substitute materials. International journal of molecular sciences, 19(10), 2893.

Shah, R., Gowda, T. M., Thomas, R., Kumar, T., \& Mehta, D. S. (2019). Biological activation of bone grafts using injectable platelet-rich fibrin. The Journal of Prosthetic Dentistry, 121(3), 391-393. https://doi.org/10.1016/j.prosdent.2018.03.027

Sheikh, Z., Brooks, P., Barzilay, O., Fine, N., \& Glogauer, M. (2015). Macrophages, Foreign Body Giant Cells and Their Response to Implantable Biomaterials. Materials, 8(9), 56715701. https://doi.org/10.3390/ma8095269

Sohn, D.-S., Huang, B., Kim, J., Park, W. E., \& Park, C. C. (2015). Utilization of autologous concentrated growth factors (CGF) enriched bone graft matrix (Sticky bone) and CGFenriched fibrin membrane in Implant Dentistry. J Implant Adv Clin Dent, 7, 11-29.

Soni, R., Priya, A., Kumar, L., \& Himanshi, Y. (2019). Utilizing autologous growth factors enriched bone graft matrix (sticky bone) and Platelet rich fibrin (PRF) membrane to enable dental implant placement: A case report. IP Annals of Prosthodontics and Restorative Dentistry, 5(1), 16-19. https://doi.org/10.18231/j.aprd.2019.005

Soni, R., Priya, A., Yadav, H., Mishra, N., \& Kumar, L. (2019). Bone augmentation with sticky bone and platelet-rich fibrin by ridge-split technique and nasal floor engagement for immediate loading of dental implant after extracting impacted canine. National Journal of Maxillofacial Surgery, 10(1), 98-101. https://doi.org/10.4103/njms.NJMS_37_18 
Research, Society and Development, v. 9, n.12, e46091211501, 2020

(CC BY 4.0) | ISSN 2525-3409 | DOI: http://dx.doi.org/10.33448/rsd-v9i12.11501

Porcentaje de contribución de cada autor en el manuscrito:

Gabriela Nathaly Berrezueta Arízaga - 50\%

David Manuel Pineda Álvarez - 50\% 